\title{
DEMENTIA CARE AND GENERAL PHYSICIANS - A SURVEY ON PREVALENCE, MEANS, ATTITUDES AND RECOMMENDATIONS
}

\author{
Jochen René Thyrian ${ }^{1}$, Wolfgang Hoffmann ${ }^{1,2}$ \\ ${ }^{1}$ German Center for Neurodegenerative Diseases Rostock/Greifswald, Germany \\ ${ }^{2}$ Institute for Community Medicine, University Medicine Greifswald, Germany
}

\section{SUMMARY}

Introduction: General physicians (GP) play a key role in providing appropriate care for people with dementia. It is important to understand their workload and opinions regarding areas for improvement.

Material and Methods: A group of 1,109 GPs working in Mecklenburg-Western Pomerania, Gemany (1.633 million inhabitants), were identified, contacted and asked to participate in a written survey. The survey addressed five main topics: (a) the GP, (b) the GP's practice, (c) the treatment of dementia, (d) personal views, attitudes and specific competences regarding dementia and (e) the GP's recommendations for improving dementia-related health care.

Results: The survey response rate was $31 \%$. In total, the responding GPs estimated that they provided care to 12,587 patients with dementia every quarter year. The GPs also reported their opinions about screening instruments, treatment and recommendations for better care of dementia patients. Only $10 \%$ of them do not use screening instruments, one third felt competent in their care for patients with dementia and $54 \%$ opt for transfer of patients to a specialist for further neuropsychological testing.

Conclusions: Four conclusions from this study are the following: (a) dementia care is a relevant and prevalent topic for GPs, (b) systematic screening instruments are widely used, but treatment is guided mostly by clinical experience, (c) attitudes towards caring for people with dementia are positive, and (d) GPs recommend spending a lot more time with patients and caregivers and provision of better support in social participation. A majority of GPs recommend abolishing "Budgetierung", a healthcare budgeting system in the statutory health insurance programmes.

Key words: general physician, dementia

Address for correspondence: J. R. Thyrian, DZNE Rostock/Greifswald, Ellernholzstr. 1-2, 17489 Greifswald, Germany. E-mail: rene.thyrian@ dzne.de

\section{INTRODUCTION}

Providing care for patients with dementia in addition to tailored support for their relatives is a challenge to the existing health care system, and its significance will increase further in the near future. Due to massive demographic changes, the health care system faces more elderly people $(1,2)$ and thus an increase in patients with age-associated illnesses. Dementia is one of the most rapidly growing age-associated conditions. This trend is likely to add to the imminent undersupply of professional caregivers, especially as the utilisation of medical care increases (1). The federal state of Western Pomerania Mecklenburg (Mecklenburg-Vorpommern, MV), Germany, is experiencing this trend at an accelerated rate due to the growing population 65 years and older and the simultaneous decrease in total population. The demographic change in MV is expected to occur faster than in any other state (3).

It is estimated that $6-9 \%$ of people 65 years and older in industrialised countries suffer from dementia. In Germany, it is estimated that 1.07 million people above the age of 60 have mild to severe dementia. The prevalence increases with age: from $0.6-0.8 \%$ for $60-64$ year-olds, to $30-43 \%$ for $100+$ year-olds (4). There are annually 250,000 incident cases of dementia (5).
A prognosis for MV from 2005 indicated that there were approximately 19,400 people with dementia and that this number would increase by $80-91 \%$ until 2020 . Although these are the most current statistics for Germany, there are limitations to the data. Some data stem from meta-analyses that combine studies with different assessment methods, sample sizes or target samples (6, 7). The most recent study by Ziegler \& Doblhammer (8) is based on the ICD-10 diagnosis of dementia in 2002 for members of one statutory health insurance company. However, it is known from prospective population-based studies that the proportion of false negatives for dementia exceeds $60 \%(9,10)$. The general physician (GP) plays a key part in providing appropriate care for people with dementia. According to the Federal Bureau of Statistics, $83 \%$ of the people 60 years and older consult their GP at least once in a quarter of year (11) and the majority of the caregivers regard the GP as the key person in managing care for dementia patients (12). Given the GP's importance, it is necessary to estimate the size of their patient population. Much of the care for dementia patients can only be provided by the GP, rendering them the key provider for primary data.

Little is known about the delivery of dementia care by GPs. While different guidelines for the treatment of dementia have been 
issued (13-15) and various screening instruments are available, the utilisation of these tools is unknown. Recent analyses have indicated that a majority of GPs in statutory ambulatory health care in Germany felt qualified to diagnose and treat dementia (16). There have also been efforts to develop instruments to assess personal views and dementia knowledge among GPs for epidemiological studies $(17,18)$. Evidence-based knowledge, however, is still lacking.

Recent studies indicate that there is a positive association between a GP's self-estimated competence and a positive attitude towards caring for patients with dementia. This indicates that training GPs in these competencies could translate into positive outcomes for dementia patients and their caregivers (16). This survey was conducted among randomly selected GPs from metropolitan areas (return rate 40\%) and GPs already participating in the German Competence Network Dementia (CND; return rate $84 \%$ ). This sampling frame yields a selection bias with an overrepresentation of GPs from the CND, which might overestimate their perceived competencies. There is also an under-representation of GPs from rural areas. Population-based data are needed to get a better estimate of competencies, attitudes and approaches for primary care of dementia patients in Germany.

Knowing the number of patients with dementia in primary care as well as the knowledge and attitudes of GP in caring for people with dementia is a prerequisite for strategically improving the health care system to meet dementia-related needs. GPs must be involved in this process as they deliver and coordinate the majority of dementia care. Moreover, GPs would be directly affected by any changes in the system.

The aim of this analysis is to (a) present data from a population-based survey among GPs about the number of patients with dementia treated, (b) illustrate the utilisation of screening instruments and guidelines in GP care, (c) describe attitudes and competencies in dealing with patients with dementia and their caregivers and (d) report recommendations to improve medical care for dementia patients from the GPs' perspective.

\section{METHODS}

\section{Sample}

Using a list of addresses for the Association of Statutory Health Insurance Physicians (Kassenärztlichen Vereinigung MV; KV) and the Medical Association (Ärztekammer MV; ÄK), 1,109 GPs in private practice were identified, contacted and asked to participate in a written survey in the whole federal state of MV starting in July 2010. A written reminder was sent to GPs who had not answered by early October and those where mail delivery had failed initially but a valid secondary address was obtained. At the termination of the survey in February 2011, 335 GP had participated, yielding a response rate of $30.5 \%$. The detailed data are given in Table 1.

No ethical approval was obtained. Participation in this survey was voluntary, and all research aims and data safety issues were described in the accompanying letter.

Approximately $57 \%$ of the participants were women. The mean age was 52.6 years, with a mean of 25.2 working years and 14.6 years working in residency. A total of $70 \%$ of the respondents were specialists in general medicine, $20 \%$ in internal medicine, $2 \%$ in surgery, $2 \%$ in paediatrics and $6 \%$ in other or multiple specialties.

\section{Data Assessed}

The survey included the following sections: (a) the GP (age, sex, education, etc.), (b) the practice (number of patients per quarter, number of people with dementia per quarter, number of people treated at home, and number of people treated in nursery homes), (c) diagnostic procedures and treatment provided (screening instruments, guidelines for care and neuropsychological tools used) (d) personal views, attitudes and competencies regarding dementia (following Pentzek et al. $(17,18)$ and Kaduszkiewicz et al. (16) and (e) the GP's recommendations for how to improve health care for patients with dementia.

The questionnaire about personal views consisted of 20 items, with 11 pertaining to the GP's personal views. The questions each focus on one of three categories: "attitude", "confidence", or "disclosure" (18). The original questionnaire differed in the response format for each category, so the format for "attitude" and "confidence" was changed. This was done to reduce confusion, with all items rated on a 5-Point Likert Scale from 1 (strongly disagree) to 5 (strongly agree). The items referring to "disclosure" remained in the original format of "never" (0), "sometimes" (1), "mostly" (2), and "always" (3).

The questionnaire addressing general attitudes, diagnostic procedures, disclosure, caregivers, self-help, guidelines and the continued education of GPs (16) consisted of 24 items. In this survey, 16 questions inquired about general attitudes (4 items),

Table 1. Number of patients (with dementia) treated per GP per quarter year in Mecklenburg-Western Pomerania

\begin{tabular}{|l|c|c|c|c|c|c|c|}
\hline Item ( $\mathrm{n}$ of GPs with informative answers) & $\Sigma$ & Mean & SD & Median & Min & Max & GP affected \\
\hline Patients treated per quarter year ( $\mathrm{n}=318)$ & 346,244 & 1,089 & 368.3 & 1,000 & 20 & 2,700 & $100 \%$ \\
\hline $\begin{array}{l}\text { Patients with dementia treated per quarter year } \\
(\mathrm{n}=327)\end{array}$ & 12,587 & 38 & 37.2 & 30 & 0 & 300 & $326(100 \%)$ \\
\hline $\begin{array}{l}\text { Proportion of number of patients treated with } \\
\text { dementia per patients treated ( } \mathrm{n}=315)\end{array}$ & & $4.8 \%$ & $10.5 \%$ & $2.5 \%$ & $0 \%$ & $100 \%$ & \\
\hline $\begin{array}{l}\text { Patients with dementia living in their own home } \\
(\mathrm{n}=323)\end{array}$ & 5,525 & 17 & 23.1 & 10 & 0 & 250 & $317(98 \%)$ \\
\hline $\begin{array}{l}\text { Patients with dementia living in nursing homes } \\
(\mathrm{n}=319)\end{array}$ & 5,455 & 17 & 37.7 & 10 & 0 & 600 & $296(93 \%)$ \\
\hline
\end{tabular}


diagnostic process ( 4 items), caregivers (3 items), self-help (2 items), guidelines and continued education ( 2 items) and competence (1 item). "Disclosure" was excluded because it had been assessed by the previous survey (18). We changed the response format from a 6-Point Likert scale to 5-Point Likert scale from 1 (strongly disagree) to 5 (strongly agree) to maintain consistency across the surveys.

We calculated the mean agreement in responses for each scale, with 1 indicating a low agreement and 5 the highest possible agreement.

The GPs were asked to provide recommendations for improving dementia care by rating each item in a list of 16 potential actions on a 5-Point Likert scale from 1 (no action needed) to 5 (action strongly recommended). The results are presented as the mean agreement among GPs per item and the percentage of all GP rating the action with 4 or 5 .

\section{Analyses}

The GPs provided descriptive statistics about the dementia patients who were treated. These include the total number of patients each GP treated $(\Sigma)$ and the mean, median, range, and standard deviation (SD) of people treated by each patient category. We also calculated the percentage of GPs presently caring for dementia patients as the ratio between the number of GPs treating at least one patient with dementia and the number of GPs responding to the survey. Ratios between patients with dementia and total patients per GP were also calculated.

The GPs were asked whether they use guidelines to treat dementia patients and which guidelines they use. Each guideline was presented separately so multiple guidelines could be selected, if applicable. The same procedures were used for screening instruments and neuropsychological tests. Only currently available dementia guidelines were assessed in this survey $(14,15,19)$ with the additional option to specify "clinical experience" or "individualised guidelines". The screening instruments assessed were the ones currently recommended (20-22) along with two additional categories, "others" and "none".

\section{RESULTS}

\section{Number of Patients with Dementia Treated by GPs}

The median number of patients treated by each GP was 1,000 per quarter year. This number ranged from 20 to 2,700 in our sample. The mean of 1,089 patients had a standard deviation of 368 , indicating that the number of dementia patients treated varied considerably between GPs. Nearly all responding GPs (99.7\%) were treating patients with dementia, with an average of 38 patients per GP. This number varied from 0 to 360 per GP per quarter year. On average, dementia patients made up $4.8 \%$ of the GPs' total patients, with the median being $2.5 \%$. Each GP cared for an average of 17 patients at home and 17 patients at a nursing home (median of 10). In total, the responding GPs delivered care to 12,587 patients with dementia per quarter year. More detailed results are presented in Table 1.

\section{Use of Screening Instruments, Guidelines and Neu- ropsychological Testing}

The use of screening instruments, guidelines and neuropsychological tests used by the GPs in MV is illustrated in Table 2. The most frequently used test is the Clock Drawing Test, followed by the MMST and the DemTect, which combined were used by more than half of the responding GPs. Over half of the GPs indicated that they individually tailored their care for patients with dementia. Only $24 \%$ of GPs indicated the use of guidelines set by

Table 2. Screening instruments, guidelines and neuropsychological test batteries used in the GP in Mecklenburg-Western Pomerania

\begin{tabular}{|c|c|c|c|}
\hline & & $\mathrm{n}$ & $\%$ \\
\hline \multirow{6}{*}{ Guidelines ( $n=335$ ) } & DEGAM-Leitlinie & 83 & 24.8 \\
\hline & S3-Leitlinie & 74 & 22.1 \\
\hline & Evidence.net & 26 & 7.8 \\
\hline & $I Q W I Q$ & 14 & 4.2 \\
\hline & Clinical experience & 181 & 54.0 \\
\hline & Individualised treatment & 221 & 66.0 \\
\hline \multirow{5}{*}{ Screening instruments $(n=335)$} & MMST & 236 & 70.4 \\
\hline & DemTect & 186 & 55.5 \\
\hline & Clock Drawing Test & 264 & 78.8 \\
\hline & Others & 30 & 9.0 \\
\hline & None & 32 & 9.6 \\
\hline \multirow{4}{*}{ Neuropsychological testing * $(n=335)$} & GDS & 57 & 17.0 \\
\hline & Barthel-Index & 110 & 32.8 \\
\hline & ADL-scale & 19 & 5.7 \\
\hline & I rather transfer to a specialist & 180 & 53.7 \\
\hline
\end{tabular}

${ }^{*}$ Reported are only tests which were named by more than $5 \%$ of the responding GPs. 
the German Society for General Medicine (DEGAM), and 22\% used the S3-guidelines by the German Society for Neurology and Psychiatry. Some GPs conducted further systematic testing for depression (17\%) and daily life activities (38.5\%). In addition, $53.7 \%$ of GPs transferred their patients to a specialist for further neuropsychological testing.

\section{Attitudes and Competencies Regarding Dementia Care in General Physicians}

The GPs' attitudes towards the diagnosis and treatment of people with dementia were positive, with more than half of the GPs indicating high agreement to each item. However, the GPs' perceived confidence in diagnosis was rated less positively. Only $18.8 \%$ indicated that they strongly agreed when asked if they felt very confident in their diagnosis. The GPs were more sceptical in their general attitude towards dementia as well as their own role in the dementia care process. Only $2.4 \%$ indicated strong agreement with these items. There is scepticism towards the dementia detection process, with an average agreement of 2.89. The agreement towards self-help is more clearly divided among supporters $(25 \%)$ and opponents (19\%), although the GPs agreed that guidelines and continued education were needed. One third felt competent in their care for patients with dementia. The vast majority of GPs (76.1\%) disclosed the diagnosis to a caregiver, while disclosure to the patient was less universal. Only $20.1 \%$ indicated that they always disclose a dementia diagnosis, while the majority $(60.1 \%)$ reported disclosing the diagnosis only most of the time.

\section{Recommendations for Improving Dementia Care}

The GPs reached the greatest consensus in providing the recommendation to abolish the "Budgetierung". The "Budgetierung" is an economic tool imposed by the mandatory statutory health insurance in Germany. It defines a certain budget per patient per quarter that is intended to cover the work of the GP and his staff as well as all medication and therapeutic appliances. In this highly political issue, physicians argue that this budget restricts therapeutic options, while economists contend that this is a necessary tool to limit rising health care costs.

The GPs also highly recommended to be able to spend more time with his/her patient and caregiver, with $86 \%$ of all respondents agreeing that action is needed (4 and 5). Additional strong recommendations, indicated by a mean greater than 4 , included need for social participation outside the health care system and public education. Increasing patient education and financial support for caregivers was recommended least frequently by the GPs, both of which were supported by less than half of respondents. The low importance given to promotion of patient's education needs to be discussed further. The present survey did not deal with this issue. On the one hand this result could reflect that people with dementia are perceived as not being capable to be educated or are detected in later stages of dementia. On the other hand this could reflect experiences that for several reasons (denial etc.) patients with dementia are hard to educate and that the education of the caregiver is perceived as more beneficial in the long run. However, this question remains unanswered in this study. Detailed results are presented in Table 3 .

\section{DISCUSSION}

Dementia care is a prevalent and relevant issue for GPs in Germany. All but one GP participating in this survey indicated that they treated patients with dementia. However, the proportion of patients with dementia out of the total GP patient population indicates that dementia is not one of the most frequent diagnoses, especially compared to obesity, cardiovascular diseases or diabetes. Approximately half of dementia patients treated by GPs still live at home, while half are cared for in another type of stationary setting. These heterogeneous circumstances pose a challenge for any kind of ongoing systematic education focused

Table 3. Recommendations to improve dementia care as perceived by GP in Mecklenburg-Western Pomerania

\begin{tabular}{|l|c|c|}
\hline Item & Mean & Recommended by \\
\hline Abolish "Budgetierung" & 4.40 & $82.0 \%$ \\
\hline GP needs more time for the patient/ caregiver & 4.38 & $86.3 \%$ \\
\hline Improve offers for social participation & 4.24 & $82.4 \%$ \\
\hline Improve education of the social environment & 4.13 & $76.6 \%$ \\
\hline Increase information for caregivers & 3.97 & $72.3 \%$ \\
\hline Community must be better prepared & 3.93 & $69.3 \%$ \\
\hline Improve reimbursement for treatment & 3.91 & $67.6 \%$ \\
\hline Increase cooperation with other physicians & 3.88 & $69.5 \%$ \\
\hline Improve qualification of professional caregivers & 3.85 & $67.1 \%$ \\
\hline Integrate relatives more in therapy and care & 3.83 & $65.8 \%$ \\
\hline Increase cooperation with inpatient setting & 3.83 & $65.1 \%$ \\
\hline Increase cooperation with ambulatory treatment & 3.80 & $66.0 \%$ \\
\hline Increase availability of low-threshold helps & 3.73 & $61.0 \%$ \\
\hline Increase patient education & 3.42 & $45.9 \%$ \\
\hline Increase financial support for caregiver & 3.37 & $44.6 \%$ \\
\hline
\end{tabular}




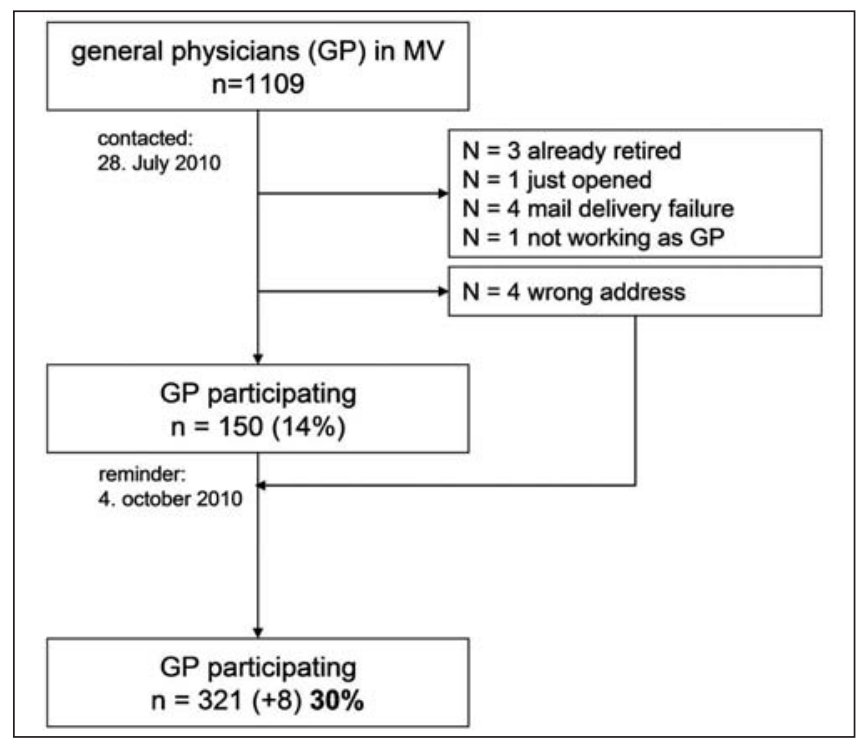

Fig. 1. Sample description.

on dementia. This challenge might additionally be reflected in the fact that guidelines are rarely used by the GPs. The guidelines presently available may be too specific for dementia or may not reflect the real practice of GPs. On average, only 17 patients living at home were treated in each practice, and these patients may be very diverse in their health and personal circumstances. This may lead GPs to rely on their clinical experience more than guidelines. However, this does not necessarily reflect a general negative attitude towards systematic approaches because most GPs also reported using valid, systematic screening instruments in their daily practice.

In this sample of GPs, attitudes towards caring for people with dementia were generally positive. This is consistent with previous descriptions of GPs being dedicated to and concerned with caring for their patients with dementia (16). The results show, however, that there is a need to improve the detection process and increase the inclusion of caregivers, with very few respondents agreeing that either one currently occurs at a sufficient level. In addition, guidelines and GP education should be improved because there is a lack of consensus among GPs that the currently available tools are working.

The GPs participating in this survey would improve the care for people with dementia and their caregivers by spending more time with them and integrating them into social environments. The GPs were supportive of the recommendations presented in this survey. However, the GPs tended to believe that improving qualification, cooperation, reimbursement, and availability of formal help were not as important as improving societal understanding and the quality of patient care. Health policies and operational health research should take this finding into account when considering how to improve dementia care. The most strongly supported recommendation, however, was the abolishment of the "Budgetierung", which has economic repercussions.

There are limitations to this survey that may affect the interpretation of the results. First, the limited participation (low response rate) might have caused a bias in the selection of the sample. This may be a threat to the generalisability of the results to all GPs in MV or Germany. Only a third of the GPs in the federal state of
MV answered the survey. This might lead to an over representation of GPs that were interested in the topic of dementia, thereby overestimating the number of patients with dementia treated by GPs. This could also present a skewed picture of treatment, attitudes and recommendations supported by GPs. However, a $30 \%$ participation rate in population-based survey in the field of primary care is very common. A second weakness is that the ratings represent the GPs' perceptions and judgements. There is no objective data that validates the reported number of patients treated with dementia. Previous efforts have used secondary data. To obtain valid data, a systematic screening for patients as well as an observation of treatment in the GP practice, would be necessary, and this would be a very costly process.

\section{Key Points}

There are four conclusions that can be drawn from the data presented in this article:

1. Dementia care is a relevant and prevalent topic for GPs in Germany.

2. Systematic screening instruments are widely used; however, the treatment of people with dementia is guided mostly by clinical experience.

3. Attitudes towards caring for people with dementia are positive, which is consistent with previous research that GPs are dedicated to and concerned with caring for their patients with dementia.

4. The highly supported recommendations for improvement include more time for patients and caregivers and more support in social participation of the patients. The majority of GPs also recommend abolishing the "Budgetierung".

\section{Conflicts of interest}

None declared.

\section{REFERENCE}

1. Siewert U, Fendrich K, Doblhammer-Reiter G, Scholz RD, Schuff-Werner $\mathrm{P}$, Hoffmann W. Health care consequences of demographic changes in Mecklenburg-West Pomerania: projected case numbers for age-related diseases up to the year 2020, based on the Study of Health in Pomerania (SHIP). Dtsch Arztebl Int. 2010 May;107(18):328-34.

2. Doblhammer G, Muth E, Kruse A. Life expectancy in Germany: trends, prognosis, risk factors and impact of selected medial innovations. Rostock: Rostocker Zentrum zur Erforschung des Demografischen Wandels; 2008. (In German.)

3. Fendrich K, Hoffmann W. More than just aging societies: the demographic change has an impact on actual numbers of patients. J Public Health. 2007; 15(5):345-51.

4. Ziegler U, Doblhammer G. Prevalence and incidence of dementia in Germany - a study based on data from the public sick funds in 2002. Gesundheitswesen. 2009 May;71(5):281-90. (In German.)

5. Bickel H. Epidemiology of dementia. Das Wichtigste - Informationsblatt der Deutschen Alzheimer Gesellschaft. 11-7-2008. (In German.)

6. Bickel H. Dementia in advanced age: estimating incidence and health care costs. Z Gerontol Geriatr. 2001 Apr;34(2):108-15. (In German.)

7. Bickel H. Dementia syndrome and Alzheimer disease: an assessment of morbidity and annual incidence in Germany. Gesundheitswesen. 2000 Apr;62(4):211-8. (In German.)

8. Ziegler U, Doblhammer G. Prevalence and incidence of dementia in Germany - a study based on data from the public sick funds in 2002 . Gesundheitswesen. 2009 May;71(5):281-90. (In German.)

9. Villars H, Oustric S, Andrieu S, Baeyens JP, Bernabei R, Brodaty H, et al. The primary care physician and Alzheimer's disease: an international position paper. J Nutr Health Aging. 2010 Feb;14(2):110-20. 
10. Valcour VG, Masaki KH, Curb JD, Blanchette PL. The detection of dementia in the primary care setting. Arch Intern Med. 2000 Oct 23;160(19):2964-8.

11. Destatis. Data reports 2008. Bonn: Statistisches Bundesamt; 2008. (In German.)

12. Schoenmakers B, Buntinx F, Delepeleire J. What is the role of the general practitioner towards the family caregiver of a community-dwelling demented relative? A systematic literature review. Scand J Prim Health Care. 2009;27(1):31-40.

13. S3-Guideline "Dementias" [Internet]. 2009 [cited 2012 Nov 05]. Available from: http://www.dgn.org/images/stories/dgn/pdf/s3 leitlinie demenzen. pdf. (In German.)

14. German College of General Practitioners and Family Physicians. Dementia DEGAM Guideline number 12. Düsseldorf: Omikron publishing; 2008. (In German.)

15. Evidence.de. Guideline diagnosis, therapy and care in dementia, version 05/05 [Internet]. Medizinisches Wissensnetzwerk evidence.de; 2007 [cited 2012 Nov 05]. Available from: http://www.evidence.de/Leitlinien/ leitlinien-intern/Demenz_Start/demenz_start.html. (In German.)

16. Kaduszkiewicz H, Wiese B, van den Bussche H. Self-reported competence, attitude and approach of physicians towards patients with dementia in ambulatory care: results of a postal survey. BMC Health Serv Res. 2008 Mar 6;8:54.

17. Pentzek M, Wagner G, Abholz HH. The development of a dementia knowledge test for general practitioners. Z Arztl Fortbild Qualitatssich. 2006;100(4):283-9. (In German.)

18. Pentzek M, Abholz HH, Ostapczuk M, Altiner A, Wollny A, Fuchs A. Dementia knowledge among general practitioners: first results and psychometric properties of a new instrument. Int Psychogeriatr. 2009 Dec;21(6):1105-15.

19. Tombaugh TN, McIntyre NJ. The mini-mental state examination: a comprehensive review. J Am Geriatr Soc. 1992 Sep;40(9):922-35.

20. Kalbe E, Brand M, Kessler J, Calabrese P. The DemTect in the clinical application: sensitivity and specificity of a cognitive screening instrument. Zeitschrift für Gerontologie \& Psychiatrie. 2005;18(3):121-30. (In German.)

21. Sunderland T, Hill JL, Mellow AM, Lawlor BA, Gundersheimer J, Newhouse PA, et al. Clock drawing in Alzheimer's disease. A novel measure of dementia severity. J Am Geriatr Soc. 1989 Aug;37(8):725-9.

Received December 15, 2011 Accepted in revised form September 5, 2012 